\title{
Analysis of the Approach on Usability and Ergonomics in Handling and Transporting Packaging
}

\author{
Marília Colozio Favaro, Adriana Yumi Sato Duarte and Franco Giuseppe Dedini \\ Laboratory of Integrated Systems, Faculty of Mechanical Engineering, State University of Campinas, Sao Paulo 13083860, Brazil
}

Received: April 15, 2014 / Accepted: May 13, 2014 / Published: June 25, 2014.

\begin{abstract}
The purpose of this research is to explore the principle of the man-object relationship to identify the issues shown when handling packaging and emphasizing the usability aspect. For that, the theoretical reference of the research involves the analysis of the parameters according to the aspects that involve the use interactions of packaging in agreement with the definition and scientific concepts of ergonomics, allied to the development of product projects, discussing forms, handling, load transportation and the support of the product. Therefore, improperly handling of the packaging may cause damages to the products and even potential accidents. Coding and decoding are significant to facilitate the use recognition within the individual's repertoire. The packaging, within the macro-ergonomics universe, may be the object of ergonomics, whose contribution to solve problems is related to labor activities, by dealing with packaging, transportation and load resulting from the man-object interaction. The incorrect identification of information may lead to problems of interpretation and misuse, from the handling to the disposal of packaging. For this reason, this research suggests the adjustment of the man-object interface which may lead users to make mistakes regarding use and important choices, through adequate handling actions.
\end{abstract}

Key words: Man-object, handling packaging, usability, packaging, product development.

\section{Introduction}

Ergonomics is the term that designates the multidisciplinary application of knowledge to deal with a series of cautions involving men and the inherent particularities of each task performed under labor conditions, observing the individual characteristics and limitations. For such, this study must be more broadly interpreted, not only in relation to physical effort, but in all dimensions. Only then the objective of maximizing the results of this study and minimizing effort, fatigue and potential harm to the integrity of human health will be reached.

The adjustment between object and information is a reference for the man-object interface. Non-significant codes and denominations may lead users to make mistakes regarding the use and important choices. Coding is meaningful so that there is an easier

Corresponding author: Marília Colozio Favaro, master, research fields: packaging, product development and design methodology. E-mail: mcfavaro@fem.unicamp.br. recollection and use recognition [1].

The study of the symbols and identification of the problems that may occur due to the inadequate use of packaging was based on the man-object analysis. The user's experience in relation to the object suggests principles for a good design, building a psychological method to interact with the objects, based on principles are highly inter-relational with other areas or other terms [2].

Usability is the capacity that an interactive system offers to the user, within a specific context, in order for the task to be effectively, efficiently and comfortably performed, and it determines specifications and evaluations emphasizing the performance and satisfaction of the operator/user. Partially, multidisciplinarity is essential for its correct use during the project's conception.

The packaging has a fundamental role to protect the products' quality, in addition to making their transportation feasible, in order to perfect sustainably 
the product's distribution chain. The project methodology guides this study due to the complex network of variables involved, mainly because this is a multidisciplinary theme.

The objective of this research is to show the definitions on the theme, elaborating a discussion on the different study branches involved with this concept and finally, elaborating a theoretical analysis suggesting an adjustment of the man-object interface, in which information is the reference for the non-significant codes and denomination, considering that they may lead the user to make mistakes as to use and important choices through adequate handling and transportation actions. Thus, the approach to Ergonomics is justified, especially regarding usability with the purpose of contemplating these new parameters.

The paper is organized as follows: Section 2 presents the research scenario, introducing the ergonomics and usability concepts, packaging technology and transportation; Section 3 analyses and discusses the ergonomic factors applied to the handling and transportation of packaging; and Section 4 presents the conclusions and emphasizes the importance of ergonomics for comfort and safety.

\section{Research Scenario}

The purpose of this section is to discuss the main research themes: packaging technology by function, shape and classification, the importance of the ergonomics parameters, specifically usability and handling to perform a specific task.

\subsection{Ergonomics}

Thus, the word Ergonomics comes from the Greek words ergo (work) and nomos (rules) and, in some other territories, it may be found with the denomination human factors, and it is applied to the design of machines, equipment, products, tools, systems and tasks with the purpose of improving the safety, health, comfort and efficiency of the work.
The purpose of Ergonomics is always to adapt or adjust the object in the best possible way to human beings in general, in relation to safety, comfort, and use efficiency or operational handling of the objects, in activities and human tasks [3].

It can be said that Ergonomics is the source of usability, since the purpose of both is to provide efficacy, efficiency, well-being and health to the user by adapting his/her way of thinking. The Brazilian Association of Ergonomics (ABERGO) [4] adopts the definition of "Ergonomics as the study of interactions between people and technology, the organization and the environment, aiming at interventions and projects to improve, in an integrated and non-unrelated manner, the safety, comfort, well-being and efficiency of human activities".

Thus, Wisner [5] defines Ergonomics as "a set of scientific knowledge related to humans and necessary to create tools, machines, and devices that may be used with the utmost comfort, safety and efficiency". For Grandjean [6], Ergonomics may be defined as "the science of configuring work adapted to humans". In the beginning, the configuration of tools, machines and the work environment was considered.

According to IEA (International Ergonomics Association [7]), Ergonomics (or human factors) is the scientific discipline related to understanding the interactions between humans and other elements or systems, and to the application of theories, principles, data and project methods, in order to optimize the human well-being and the global performance of the system.

Among the characteristics of Ergonomics, we may mention three types of approach: Physical Ergonomics, which involves several human characteristics; Cognitive Ergonomics, which involves cognition processes; and Organizational Ergonomics, which involves the social-technical elements.

However, the basic objectives of Ergonomics are the ones that encompass health, safety and satisfaction of the worker, which is the consequence of an 
adequate planning and work organization.

Human posture is the decision to keep the body in a desired position related to biomechanical matters [3]. General biomechanics studies the interactions between work and man under the perspective of skeletal muscle moves. Iida [8] states that Occupational Biomechanics is part of General Biomechanics and, thus, Gomes [3] emphasizes that it studies the interactions between man and work under the perspective of skeletal muscle moves.

Therefore, the activities performed at work may be shown as follows:

- Static work - the one that requires continuous contraction of some muscles, in order to maintain a certain position;

- Dynamic work - the one that allows for alternate contraction and relaxation of the muscles.

The force applied may occur in several different ways, such as pushing, pulling, squeezing and pressing among others, which will depend on the work performed [8]. The number of people with spinal problems is increasingly higher. Several studies in many countries have shown the relationship between the handling activities and manual material handling and the incidence of a large number of accidents and osteoarticular lesions, mostly in the lumbar region [6].

Whenever possible, the control moves must follow the natural moves that are more easily performed by humans. Based on the Ergonomic principles, machines and tools are considered as an extension of men. The man-object interaction contributes to reduce mistakes, ergonomic stress and accidents in order to improve the performance of the system in which it is inserted. All the moves made by the human body with the purpose of transmitting some form of energy to the machine (usually performed with the hands and the feet) are called control movement. Therefore, the hands must make paced moves, through a curved and continuous orbit, avoiding sudden changes and harsh interruptions [8].

Ergonomics makes use of Biomechanics which consists of analyzing the mechanical bases of biological activities directed towards the human muscle-skeleton system in order to study the human body's posture at work, application of force and handling.

The movement of body segments is defined as "the angular displacement around an imaginary rotational axis that goes through the articulation to which the segment is connected” [9]. For lifting and manual material handling, the weight on the spine must be applied towards the axis and close to the body, in order to hinder perpendicular components from appearing as shown in Fig. 1.

Load transportation may cause two types of reactions on the user: the overweight causes an excessive physiological load on the spinal muscles and the lower limbs; and the postural stress, caused by the body's contact with the load. Consequently, pain, discomfort and ergonomic stress are caused. The product development together with Ergonomics looks for efficient alternatives to transport load that would reduce energy use and muscle-skeleton problems. Adopting alternatives such as keeping the load close to the body, adopting an adequate value for the unitary load, using symmetrical loads, projects such as adequate handling, teamwork, defining the path to be

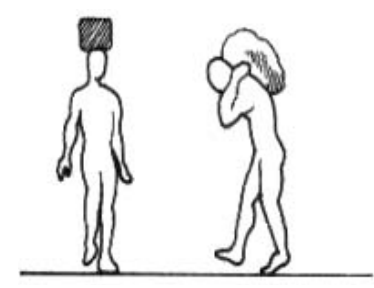

(a)

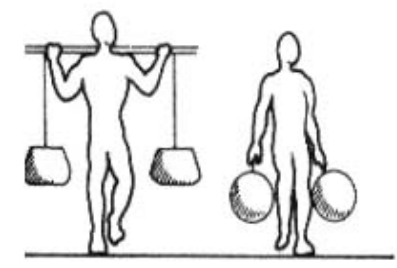

(c)

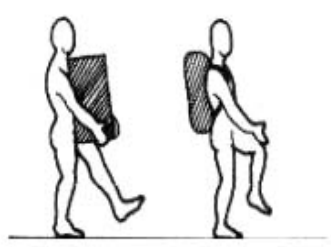

(b)

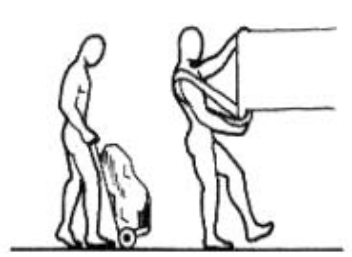

(d)
Fig. 1 Manual handling: (a) vertical load; (b) close-to-body load; (c) symmetrical loads; and (d) use of auxiliary means [9]. 


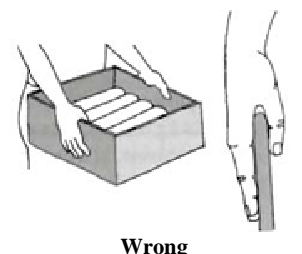

(a)

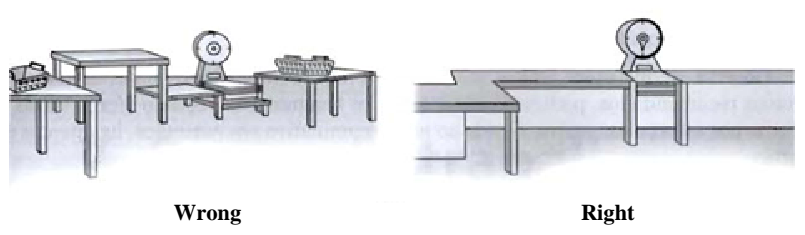

(b)

Fig. 2 (a) Material handling; (b) unevenness at the work area [8].

taken, leveled floors, use of mechanical carriers (Fig. 2), may avoid excessive effort of the dorsal muscles among other traumas caused by impact.

The operator is subject to actions such as lifting, bending down, pushing, pulling, holding and transporting load to perform a certain task. The elements that constitute this manual material handling system require the operator (defined due to his/her physical, sensorial and psycho-motor characteristics); the material (load), which has mechanical and geometrical characteristics, handling and stability, task and work space divided into geometric, temporal, complex and environmental characteristics; and work practices to be performed, which may be individual, organizational and administrative.

There are general handling classifications that are divided into two basic types [8]:

- Fine manual handling or precision handling: the moves are transmitted by the fingertips, while the palm of the hand and the fist remain still. It is characterized by great precision and speed with little force transmitted during the moves;

- Manual handling or force handling: the moves are performed with the center of the hand, keeping the fingers still and with the grasping function, while the moves are performed by the fist and arm. It transmits stronger forces, with lower speed and precision than in fine handling.
The handling design is completely relevant for the performance in the man-object system, considering the handling types. For fine manual handling, smaller shapes than the ones used for manual handling are needed. These shapes are classified into geometric and anthropomorphous.

The geometric handling is characterized by regular shapes, such as cylinders, spheres, cones and others. Since they differ from the human anatomy, they have little contact surface with the hands. Due to the use flexibility, which allows handling variations, it is easy to adapt to the variations in anthropometric measurements. However, tensions are concentrated in certain parts of the hand, interfering in the force transmission and making it hard to perform the move. Despite being less efficient, this type of design works better if applied to operations that do not require major force.

In the anthropomorphous handling design, the surfaces are round-shaped, adjusting to the hand anatomy due to the fitting depressions or saliences, which are referred to as “anatomical”. It shows greater contact surface and handling stability in relation to geometric handling, transmitting greater force and concentrating less tension, but it is ergonomically stressful if used during long hours due to the limited position. Therefore, this kind of design shows a better performance when used for short-term tasks, which require little moves, need greater force, as well as when the users show similar anthropometric measures.

The object's superficial finishing may interfere in the handling and task performance, since each type of handling (fine manual handling and manual handling) requires an adequate surface, which, otherwise, may be harmful, since pressures are concentrated on those spots. Data show that the use of "manual tools" is related to cumulative trauma on the users' hands and forearms [8]. A diagram shows the painful areas of the hands, fist and forearms, according to Fig. 3.

In order to avoid any type of ergonomic stress when performing the work, the tool must be adequately 


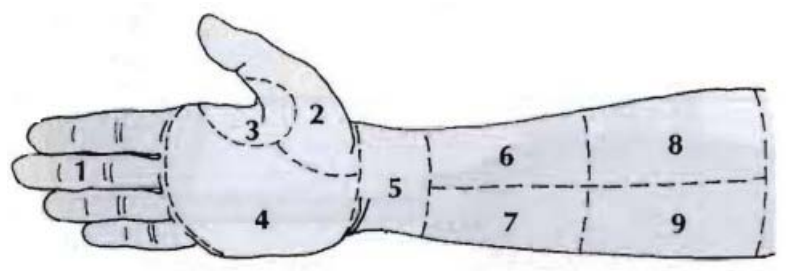

Fig. 3 Diagram to survey the painful areas when using manual tools [8].

chosen, based on its functionality, and they must be selected according to the task to be performed, followed by the ergonomic characteristics that ensure the operator's comfort and safety. Such characteristics depend on two factors [8]:

(1) Handling characteristics: the differences in the handling methods must be considered, the transmitted moves (force, speed and precision), possibility to use both hands and adjustment for left-handed people. These measures allow the reduction of tension on the hands, as well as a better handling design and greater handling diameter eliminating angular surfaces;

(2) Center of gravity: the tool's center of gravity must be located as close as possible to the center of the hand, allowing better motor control in order to reduce moves and consequences, muscle efforts and energy use during the operation.

A large number of parameters are observed, trying to combine the advantages and disadvantages of each one, in order to soften the handling rigidity and increase the contact area.

\subsection{Usability}

Oftentimes, incompatibility problems on a same product cause all the improvement to be left aside, forcing the user to keep on using methods that he/she already knows. However, the user needs autonomy to demand functionality and make easy use a priority.

Usability depends on the specific characteristics of the object's interface and the user in the determining sense of the objectives applied to the use situation. The same interface may be satisfactorily interpreted for old users, but it may not be as satisfactory for new users. The essence that designates the use is in establishing a relationship between interface, object, user, task and environment [1]. The current concept is excessively involved to the cognitive aspects of the task, focusing on experiences and individual characteristics, abilities and emotional perception, which approaches a need to enrich a usage concept [10].

Partially, the social and behavioral sciences are fundamental in order to make use of usability for the design's conception, where four principles are used, involving the user and the task requirement. According to ABNT (the Brazilian Association of Technical Standards [11]) NBR 9241-11, usability is the "measure according to which a product may be used by specific users to reach specific objectives effectively, efficiently, and satisfactorily in a specific use context”. To better clarify, the concepts described are defined as:

- Efficacy-accuracy and completeness with which users achieve specific objectives;

- Efficiency-resources spent regarding the accuracy and reach with which users achieve objectives;

- Satisfaction-lack of discomfort and presence of positive attitudes towards the use of a product;

- Usage context-users, tasks, equipment (hardware, software, and materials), and the physical and social environment in which a product is used;

- User - a person who interacts with the product;

- Objective-intended result;

- Task - set of necessary actions to achieve an objective.

Therefore, what the standard dictates on the use of a certain objective (or system) can be noticed, taking into consideration its relationship with the user. Within this prism, it can be said that the term usability comprehends any relationship between the user and the environment, and the main subject matter is the interface between them and any of its consequences on the person, the object and/or the environment. 
Usability, when considered as a usage quality, is not only an aspect of the product's quality, but a consequence of the interaction between user and the product when performing a task in a certain context [12].

In the beginning, the study focused on the machine and the worker should adjust to it. The workers were selected and qualified according to the specific demands and characteristics of the machines, making the worker engage into long periods in the learning process.

Afterwards, in relation to the problems detected by human mistakes, a study that focused on the man was established. No attempt was made to modify the machines, taking into the limitations of men consideration. Finally, a system was conceived in which the man-machine interaction, specifically the man-work interaction, is taken into consideration.

Considering the orientations of the ergonomic parameters for load mobilization, it was shown that manual work may cause severe health problems due to the operator's bad human posture. However, alternatives were created to relief the load's weight on the spine, that is, wheel carts, stackers, load unification packaging; these are accessories that intend to relief the excessive weight on the user, in addition to optimizing the process. However, even with this type of mechanical work, human posture and handling actions must be applied, since the static work may be ergonomically stressful, and a bad sitting posture may cause irreversible damages to the spine. Thus, attention must be paid to the ergonomic parameters, in order for the product design to abide to the standards, offering better work conditions and comfortable safe and efficient man-object interaction.

Within the context of the man-machine system, Chapanis [13] points out that people are used or involved in any system of equipment, because the systems of equipment are at all times elaborated according to some human objective. They are there to meet a certain human need. In addition, the systems are planned and built by human beings. Human creatures handle them, oversee them, observe how they work and take care of their maintenance. The man-machine system is the system of equipment in which at least one of the components is a human being, who acts or intervenes in the operation of mechanical components of the system at all times.

In man-machine systems, people are used or involved in any systems of equipment, considering that they are at all times elaborated according to some human objective. They are there to meet a certain human need, and are developed from one human being to another. "It is the system of equipment in which at least one of the components is a human being, who acts or intervenes in the operation of mechanical components of the system at all times" [13]. Under this perspective, a product that is considered as non-usable within a system may be used for another, since usability does not depend only on the characteristics of the product, but on the user/environment relationship [10].

\subsection{Packaging}

The term "packaging", which comes from the verb "to pack", "the act of conditioning (good or objects) into packaging, packs and boxes, etc., in order to protect them from risks or facilitate their transportation” [14], has as its primary role protection and transportation. As humans became aware of their basic needs to eat, store and preserve food for a longer time and, as the distance between the supply sources increased, reaping and hunting were no longer enough, they needed to store and transport [15].

The Brazilian Ministry of the Environment [16] on DL 366-A/97 defines packaging as "all and any products made from materials of any nature used to contain, protect, transport, handle, deliver, and present goods, both as raw materials and as transformed products, from the producer to the user or consumer, including all the "disposable" alternatives used for the same purposes”. 
The Brazilian Packaging Association [17] determines that packaging is every container or wrapping that stores products temporarily, individualizing or grouping units, with the main role of protecting them in order to extend their shelf life, enabling their distribution, identification and consumption. Understanding packaging as the creation process, its efficiency and performance are verified according to the product's success, which implies the adversities of creation, interpreted as a technical object, with a particular role and characteristics [18].

However, the packaging is a thin line between the product's design and the graphic design, since both aspects are complementary and merged into three different levels: primary, secondary and tertiary [14] as represented in Fig. 4. The primary packaging design is directed towards the consumer, has a market appeal, good shelf arrangement and protects the process [21]. It is responsible for preserving and maintaining the product.

In the case of the secondary packaging, cardboard boxes, bags or casks are usually focused on logistics to offer greater handling efficiency and used to group products which are called secondary packaging. The purpose of this kind of packaging is to avoid damages upon the product's handling and storage [21].

In the tertiary packaging, the design is developed aiming at the collective transportation of the previous packaging, focusing on the protection against damages caused by the journey, prioritizing the quality of the product that will get to the end consumer. This type of packaging is usually made from a coarse material, corrugated cardboard, plastic and wood. For this type of packaging, the visual astatic is not a relevant factor since it does not get to the consumer.

The combination of these levels, due to the characteristics of the products, applicability and marketing image, guides the parameters of the product's development. The importance of this segment is due to the different roles of each one of them. The primary packaging must protect and

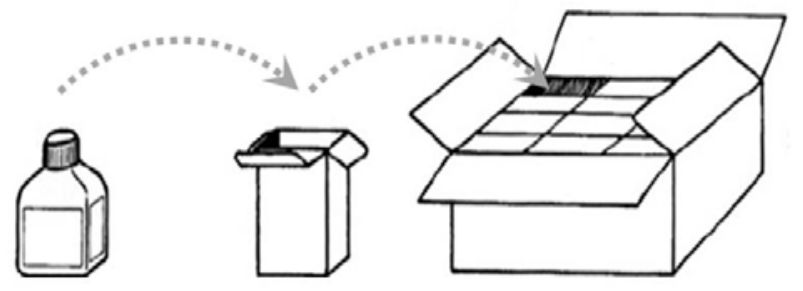

(a)

Fig. 4

(b)

(c) packaging [14].

preserve the chemical and physical integrity of the product, minimizing the absorption of gases (oxygen, carbon dioxide), humidity, odors and light among others; secondary and tertiary packaging must also help in the transportation, grouping them or optimizing mechanical aspects, protecting the product from shocks, vibrations, falling and pressure, etc., and they oftentimes work as a reinforcement [14].

The means of transportation, the level of vibration suffered during the displacement and handling of the product, the stacking height, humidity and time of stocking, among other factors, are cost-efficient, since when the lowest possible amount of material is used, the costs are reduced, increasing the number of products transported at once, and restricting investments on transportation and storage. A more resistant packaging reduces losses due to damages and stability, and offers a safer stacking. These measures are relevant considering ecologic factors, minimizing the post-use tear.

\subsection{Product Design Methodology}

In product design, information such as the product's stability and the degree of frailty or sensitivity is essential to define the level of protection the packaging will provide, considering the mechanical, chemical and electric stability.

The impacts and mechanical vibrations occurred during the transportation and shifting of the products are the main responsible factors for the damages incurred. That includes manual or equipment-based shifting, such as through conveyor belts, carts, stackers and lifters, etc., requiring adequate packaging [19]. 
The packaging development may be referred to as the act of going through a path established by the project's methodology, meeting the packaging's specificities in relation to the other industrial products, and it must be considered as a whole, that is, the context in which the product is inserted is a relevant factor for the product's development [19, 20].

In order to develop the packaging's design, the needs and simultaneities between the product's design process and the packaging's design process must be integrated. In order to develop new designs, the model is previously filled with current information on technologies, marketing researches, information products and environmental impact processes, materials, competitive intelligence, evaluation of the packaging' life cycle, in order to meet the environmental and strategic goals established [22].

The product's development is a multidisciplinary process and, as such, it needs different perspectives and expertise in order to be complete. Engineering conceives the product from a technical-physical system, with an efficient and safe operation. The design understands that the product has different roles and therefore, its good operation depends on their interactions. The design, as opposed to the common sense, is not only related to the aesthetic and symbolic aspect [23].

Another key factor for the process of developing a product is the methodology and tools used. The discussion concerning a universal definition for the term "product design methodology" shows that the acquired meaning depends on the author and the applicable area; it is generally related to the process of directing resources both financial and human to meet the defined goals.

By systematizing and describing a methodology, Asimow [24] started a discussion on the theme, and it opened a precedent for other authors to change and adapt the methodology according to the existing needs during each period, as shown in Fig. 5.

\subsection{Moving Packaging}

The objective of the design of a packaging made for
Primary phases of the project

- Phases I -Feasibility study

- Phases II - Preliminary design

- Phases III-Detailed study

Phases related to the production/consumption cycle

- Phases IV-Production planning

- Phases V-Distribution palnning

- Phases VI-Consumption planning

- Phases VII-Disposal planning

Fig. 5 Methodology [24].

transportation is to protect the product during transportation against external agents, such as mechanical shocks, dust and humidity, etc., and add several unities making the transportation, handling and storage easier.

Some aspects must be considered when specifying the packaging for transportation, such as [19]:

(1) the total weight must be adequate to be carried by one person;

(2) using closure systems that inhibit the opening as a safety measure to prevent theft;

(3) use of details that assist in opening the packaging at the point of sale.

These recommendations facilitate the use by consumers, who demand improvements on the design of packaging, such as using details that make it easier to open them. The type of material and calculating the packaging's resistance refer more to its resistance upon handling; therefore, each product has a type of specific packaging according to the context in which it is inserted. In large volume productions, the packaging need to be mechanically handled, considering as design requirements the material, tolerances, the closure system, and internal accessories.

The packaging for frail and hazardous products needs a more robust design. In the case of frail products, especially those that add value, the primary packaging must be reinforced to avoid the use of an extra packaging, exclusively for transportation, since, in addition to affecting the aesthetic; it increases the 
product's total cost. To transport hazardous products, the packaging must meet the national and international safety standards that define risk levels, material specifications and tests for approval.

The packaging that protects the product during the different transportation methods joins it from the factory up to the end user or distribution center. During the transportation, unifying the packaging involves the concept of packing or arranging several volumes of goods to create a since standardized "unit" in order to be mechanically moved along the process, serving as the basis for a chain constituted by an integrated system involving packaging, shifting, storing and transporting materials. Thus, it saves more time during displacement, in addition to protecting the product, since it is possible to see whether the volume was violated. The most used unification method is palletization, which consists of arranging upper and lower boards, connected by ring or spiral nails, adding glue (optional) to offer greater fixation; the pallets must be well aligned in order not to deform the cardboard packaging, as seen in Fig. 6. Pallets must be standardized according to the project's specifications.

Another unification method used is the slip sheets platforms, used as an accessory for the stacker during the transportation. With a solid or corrugated cardboard base and two lids measuring about $7 \mathrm{~mm}$ thick, the space taken by the packaging is minimal, and they offer another advantage which is the reduced cost in relation to any type of pallet. For this system, a stacker with a special accessory or adequate machines must be used, according to Fig. 7 .

The main characteristic of singulators is the repetitive use (except for the one-way ones), and these devices allow carrying and lifting loads through self-bearing fittings. The main unification method used by industries and warehouses is palletization, due to the pallets' transportation flexibility in automotive vehicles, airplanes, ships, railways and containers [25]. Other methods that are mostly employed for unitization are pre-language means, used for sacks, which consists

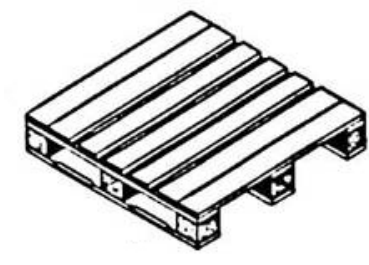

(a)

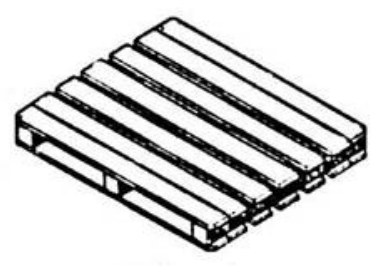

(b)

Fig. 6 (a) Four-way entry simple pallet and (b) two-way entry pallet with lids [19].

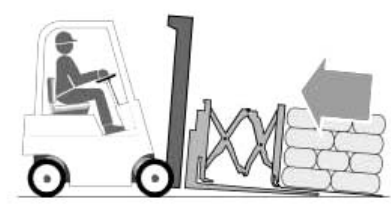

(a)

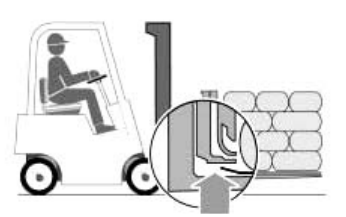

(b)

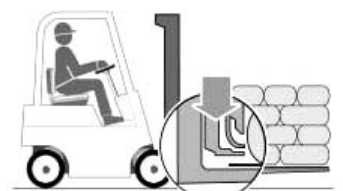

(c)

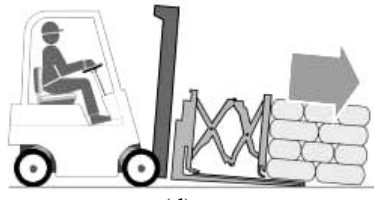

(d)

Fig. 7 Moving consolidated load units, such as slip sheet [19].
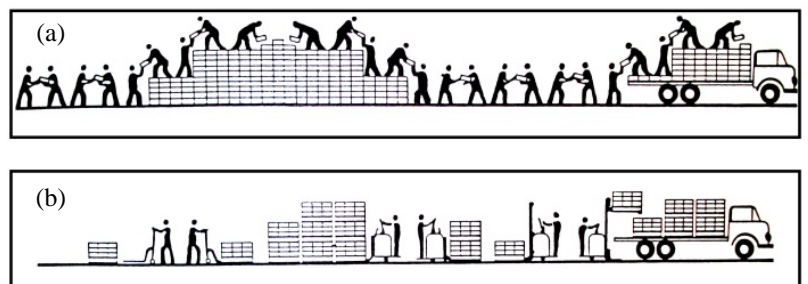

Fig. 8 (a) Manual vs. (b) mechanical transportation [25].

in tying up an object or set of objects, involved by nets or belts with lifting handles, and load self-unitization by strapping, block-stacking or bundling. Among the methods used to promote load stability during transportation or stacking, a highlight is the use of lifting devices introduced beneath the load, load compression between two surfaces and load suspension.

Fig. 8 represents the use of the unified method in comparison to the individual load handling system, showing the characteristics of each application.

The advantage of using the load unification method together with the shifting and stocking system is that it makes it easier to stack loads on top of each other,exploring the tridimensional plan by reaching considerable heights. In addition, it offers 
cost-efficient benefits for the manufacturers, shippers and consignees (facilitating price quotation) and transportation companies by increasing the operational speed of the transportation vehicle and the productivity and reducing the load shifting costs. Other advantages of applying the system are the reduced damages to the load, lower transit time and incidences of theft and reduced stock. However, it has disadvantages when it comes to controlling and returning the empty singulators.

\section{Theoretical Analysis of Usability and Ergonomic Factors Applied to the Handling and Transportation of Packaging}

For almost all operators, using mechanical transportation and packaging during transportation means no longer having to perform a dynamic work, which requires daily physical efforts when handling these objects. A few of them, however, know that a smooth-surfaced packaging, burred texture, overweight of the packaging exceeding the advisable limits, or even a stacker's seat may lead to discomfort ad even physical problems.

Several problems may arise when adequate tolls and objects are not used according to the characteristics of each person. There are several items that must be adjusted to each person, including:

(1) Chair: it must have a high backrest with arm support at the same height of the machine's panel. It needs to be precisely positioned on the lumbar curvature, supporting the spine;

(2) Height of the transporting cart: it must have an independent height regulation. The elbows must be at a $90^{\circ}$ angle in relation to the body;

(3) Workbench unevenness: the upper part must be at the same height than the eyes at a minimal distance of one arm in order to make the head to be vertically positioned and avoid irregular surfaces that force the user to move the legs. Instead, he/she should be positioned in order to be only able to rotate the body around its own axis;
(4) Shape of the packaging: surfaces must offer a steady handling to prevent the operator from using a handling method that uses excessive weight;

(5) Packaging stacking: packaging stacking must be avoided in manual handling due to spinal overload; the ideal scenario is using mechanical transportation.

The problems with packaging handling are not only related to bad human posture or with the use of inadequate equipment. The type of task and the process performed by the operator must also be taken into consideration. Overly ergonomically stressful and long tasks that require concentration to be performed reduce the operator's attention and result in physical and mental fatigue.

Several problems may arise when the work environment specifications are not being followed:

(1) Fatigue: reduced performance and work capacity. It is caused due to a change in the properties of the muscles, local intoxication or due to long and uninterrupted work hours;

(2) Backache: caused by the use of overweight and tools those are not adapted to the person. Na inadequate chair is the main factor that causes backache. The chair's backrest needs to be precisely positioned on the lumbar curvature, supporting the spine. The backrest must also be flexible in order not to allow the operator to slip backwards;

(3) RSI (repetitive strain injury): caused by the excess of repetitive movement, going back and forth through the same path, incorrect handling, machine control panels, and mainly when the load is not being transported using a correct posture. In some cases, RSI may be restricted to the affected limb, usually hands and arms. In an earlier phase, inflammation occurs, requiring immobilization and adequate medication. Depending on the quality of the resting period which should last for some months after the patient has been mobilized, he/she may or may not be able to get back to work. Some workers experience pain only five minutes after resuming the work they used to do, other take hours, and other months. 
Therefore, in order to analyze the task detailing the work procedures to make it efficient, some questions should be considered:

- Which actions are employed on the interface of the work tools and by humans?

- How do they work to reach their goal?

- Which situations must be foreseen whose use may cause risks?

- In which physical (spatial and temporal) conditions the operator and his/her instruments are involved?

- Which psycho-social relationships are there on this man-equipment/object interface in relation to shape, color and texture?

- Task description.

Questioning and surveying these data is completely relevant to develop new packaging and instruments to facilitate transportation in order to offer greater comfort for the operator, avoiding ergonomically stressful and repetitive moves, as well as energy use that may cause discomfort and severe diseases with time. In order to make the workers execute their activities, they perform varied types of body moves required by the actions, involving all of their limbs (speed, force, precision and duration); and the characteristics and types of controls used on the equipment (buttons, levers, wheels and pedals).

According to Ref. [8], the force may be directed towards pushing or pulling. In general, the force applied by humans to pull and push varies from $200 \mathrm{~N}$ to $300 \mathrm{~N}$. However, if the weight of the body and the strength of the shoulder are used to push, this value reaches $500 \mathrm{~N}$. In vertical reaches, the position causes ergonomic stress on the muscles of the shoulders and biceps; pain may also occur and be increases according to the time required for the exercise.

In relation to horizontal reaches, due to the distance between the weight and the shoulders, more is required from the muscles of the shoulders. In this position, as well as in the vertical position, the arms have to overcome certain resistances, and that is when ergonomic stress and pains occur and these positions are held for a long time.

In relation to load lifting, some care must be taken, mainly in relation to the spine, which is usually injured when they are not observed. For example, the load on the spine must be vertically applied, avoiding bending over and keeping the load as close as possible to the body, symmetrical load and auxiliary means must also be used. Therefore, important considerations to keep the human body's integrity are necessary in order not to exceed the maximum load capacity.

\section{Conclusions}

This study may broaden the knowledge on ergonomics and its different conceptual and historical aspects, its objectives and types, and mainly the awareness as to the man-object system. Incorrect handling or excessive load transportation may cause several problems to the health of the worker and the simple user. Regarding these problems, mainly in relation to the worker who is subject to long work hours, it may be questioned whether there should be a responsible party for this worker's health and well-being, that is, whether preventing these problems is a duty of the employer, the own employee or the government.

Analyzing the harms that may be caused, it may also be considered that the most adequate solution is the prevention by observing the regulatory standards, in order to avoid the possible disturbances and misfortunes. It was shown that bad human postures are oftentimes adopted due to tools and packaging those are not adequately dimensioned.

The use of the project methodology, together with ergonomics and usability, is the basis to develop alternatives, tools and objects to assist the operator and the user when transporting and handling objects in a safer and more comfortable manner. A mistaken interpretation of the task may cause severe damages and even disabilities. Objects with no usability confuse the operator/user, inducing him/her to make a 
mistake. In the work environment, in leisure areas or even within the household, such factors are called operational risks.

It is necessary to emphasize good postural practices and the correct use of equipment, instruments and objects in real situations that require active participation, in order to obtain satisfactory results: meet the goals of Ergonomics of maximizing the comfort and safety, minimize human costs and improve the quality and productivity at work. Therefore, further researches must be made to analyze the man-object relationship, as a parameter for the engineer and the designer to develop new packaging and tools/equipment, benefiting the workers and users, assisting them in the performance of their tasks, meeting their needs and minimizing mistakes.

\section{Acknowledgment}

The authors would like to thank the Laboratory of LabSIn (Integrated Systems) from the Faculty of Mechanical Engineering of UNICAMP, the financial support from CAPES and CNPq and Espaço da Escrita (Coordenadoria Geral da Universidade/UNICAMP) for the language services provided.

\section{References}

[1] W. Cybis, A.H. Betiol, R. Faust, Ergonomics and Usability: Knowledge, Methods and Applications, Novatec Editora, São Paulo, 2007.

[2] D.A. Norman, The Psychology of Everyday Things, Basic Books, New York, 1988.

[3] J.F. Gomes, Object Ergonomics, Escrituras, São Paulo 2003.

[4] Abergo-Brazilian Association of Ergonomics Home Page, http://www.abergo.org.br (accessed Jul. 2011).

[5] A. Wisner, Inside the Job-Ergonomics: Methods and Techniques, FTD S.A, São Paulo, 1987.

[6] E. Grandjean, Ergonomics Handbook, Bookman, Porto Alegre, 1998.

[7] What is Ergonomics, IEA (International Ergonomics Association), Madison, 2000.
[8] I. Iida, Ergonomics, Project and Production, Edgar Blücher, São Paulo, 2005.

[9] R.F. Abrahão, J.M. Tereso, Dciscipline "Ergonomics applied for engineering project” 2011, Feagri Unicamp.

[10] J.M.M. Neves, Study of usability with focus in PDAs, Master Thesis, UNICAMP (University of Campinas), 2005.

[11] NBR 9241-11: Usability Orientation, Brazilian Association of Technical Standards (ABNT), Rio de Janeiro, 2002.

[12] M. Hassenzahl, M. Burmestre, F. Koller, Usability engineering: Quality doesn't happen by accident, in: Proceedings of the International Status Conference of the Lead Projects Human-Computer Interaction, Saarbrücken, Germany, 2001, pp. 99-103.

[13] A. Chapanis, The Engineering and Man-Machine System, Atlas, São Paulo, 1972.

[14] C. Negrão, E. de Camargo, Packaging Design: From Marketing to Production, Novatec Editora, São Paulo, 2008.

[15] D.S. Bolognini, Packaging, Art and Technique of a People, Toga, São Paulo, 1985.

[16] Law n ${ }^{\circ}$ 366-A/97 Diary Republic, December 20th, 1997 http://dre.pt/pdf1s/1997/12/293A03/04980503.pdf (access ed Apr. 15, 2013).

[17] ABRE - Brazilian Association of Packaging Home Page, http://www.abre.org.br/setor/dados-de-mercado/texto.

[18] L.F. Silva, The Taste of Packaging, P\&D UNICAMP, Brazil, Dec. 2000.

[19] M.A. Carvalho, Packaging Engineering: An Technic Approach the Development of Packaging Project, Novotec Editora, São Paulo, 2008.

[20] F. Mestriner, Packaging Design-Basic Course, 2nd ed., Pearson Makron Book, São Paulo, 2002.

[21] C.P. Neto, Definição de embalagem e seus tipos, http://www.administradores.com.br/informe-se/producaoacademica/definicao-de-embalagens-e-seus-tipos/2170/ (a ccessed May 18, 2011).

[22] D.Z. Bucci, D.Z. Bucci, F.A. Forcellini, Sustainable packaging design model, Springer London, London, 2007, pp. 363-370.

[23] L.B. Guimarães, Sustainable brazilian design: The project process is hampered for lack of information, in: Proceedings of $6^{\circ}$ ERGODESIGN, Bauru, SP, 2006.

[24] M. Asimow, Introduction to Design, Prentice-Hall, USA, 1962.

[25] R.A. Moura, J.M. Banzato, Packaging, Unitization \& Containerization, Vol.II IMAM, 1997. 